\title{
Electrical addressing of polymer stabilized hyper-twisted chiral nematic liquid crystals with interdigitated electrodes: Experiment and model
}

\author{
A. Lorenz, ${ }^{1, a)}$ D. J. Gardiner, ${ }^{1}$ S. M. Morris, ${ }^{1,2}$ F. Castles, ${ }^{1}$ M. M. Qasim, ${ }^{1}$ S. S. Choi, ${ }^{3}$ \\ W.-S. Kim, ${ }^{3}$ H. J. Coles, ${ }^{1}$ and T. D. Wilkinson ${ }^{1}$ \\ ${ }^{1}$ Centre of Molecular Materials for Photonics and Electronics, Department of Engineering, University of \\ Cambridge, 9 JJ Thomson Avenue, Cambridge CB3 OFA, United Kingdom \\ ${ }^{2}$ Department of Engineering Science, University of Oxford, Parks Road, Oxford OX1 3PJ, United Kingdom \\ ${ }^{3}$ LG Display, R\&D Center, 1007, Deogeun-ri, Wollong-myeon, Paju-si, Gyeonggi-do 413-811, South Korea
}

(Received 7 January 2014; accepted 30 January 2014; published online 18 February 2014)

\begin{abstract}
Electro-optic switching in short-pitch polymer stabilized chiral nematic liquid crystals was studied and the relative contributions of flexoelectric and dielectric coupling were investigated: polymer stabilization was found to effectively suppress unwanted textural transitions of the chiral nematic liquid crystal and thereby enhance the electro-optical performance (high optical contrast for visible light, a near ideal optical hysteresis, fast electro-optic response). Test cells were studied that possessed interdigitated electrodes to electrically address the liquid crystal. Based on simulations, a well-fitted phenomenological description of the electro-optic response was derived considering both flexoelectro-optic and Kerr-effect based electro-optic response. (C) 2014 AIP Publishing LLC. [http://dx.doi.org/10.1063/1.4865558]
\end{abstract}

Significant work has been devoted to liquid crystal (LC) electro-optic elements for use in applications ranging from telecommunications to display devices. Generally, such systems are based on nematic LC technology, well proven in the displays industry. The electro-optic response time of nematic liquid crystals is typically in the range of several milliseconds or longer. Such relatively slow electro-optical response is a limiting factor for applications of nematic LCs in high-bandwidth telecommunication phase-devices and in next generation display devices that require field sequential color. One important, alternative, fast-switching LC mode is flexoelectro-optic switching in chiral nematics, ${ }^{1-3}$ where two modes of operation are possible. The first one has been examined in devices with planar electrodes, where the helical axis of the chiral nematic LC is aligned parallel to the electrode planes (uniform lying helix, ULH). The fabrication of ULH devices usually involves complicated alignment procedures where thermal or mechanical treatments are combined with the application of electric fields. ${ }^{3-6}$ The second mode of operation has been examined in uniform standing helix ${ }^{7-10}$ (USH) devices with interdigitated electrodes, where the helical axis of the chiral nematic LC is aligned perpendicular to the electrode plane (Figure 1). In these devices, uniform LC alignment can be easily achieved by using simple alignment layers, which do not necessarily require rubbing. In USH-devices, high optical contrast can be achieved if the helical pitch $p$ is adjusted to small values in order to avoid selective reflection of visible wavelengths and reduce the response time..$^{8-11}$ Due to their small helical pitch, such hyper twisted chiral nematic liquid crystal displays show no birefringence for visible light that propagates parallel to the helical axis. Thus, an extinct optical state can be achieved in the field-off state by assembling the device in-between crossed polarizers (Figure 1).

\footnotetext{
a)E-mail: alexander.lorenz@online.ms
}

In contrast to the field-off state, the LC layer will show birefringence for visible light if the device is electrically addressed. If so, the LC layer will act as an uniaxial medium $^{12}$ for light that propagates perpendicular to the plane of the device (parallel to the $y$-direction). However, addressing with interdigitated electrodes can also cause unwanted, almost irreversible textural transitions of the chiral nematic LC, because it also causes high out-of-plane components of the addressing electric fields. Anyway, the performance of electro-optical devices based on chiral nematic LCs can be enhanced by polymer-stabilization in various ways. ${ }^{13-22}$ In the present work, a polymer-stabilized USH-device was systematically studied to effectively suppress unwanted structural transitions, which enhanced the electro-optical performance. The studied device showed fast switching

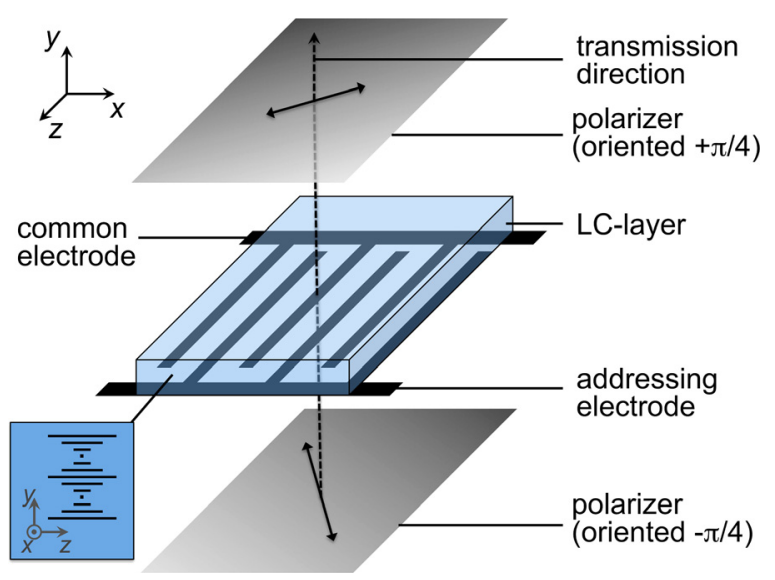

FIG. 1. Schematic of the LC test cells and polarizer configuration used in the electro-optic measurements. The planar test cells were oriented parallel to the $x z$-plane and the interdigitated electrodes were oriented in $z$-direction. The polarizers were carefully adjusted to provide transmission of linear polarized light with the electric field vectors oriented at angles of $\pm \pi / 4$ with respect to the $x$-direction. A schematic of the helical director field of the LC (one pitch, helical axis parallel to $y$-direction) is shown as inset. 
(response times $<300 \mu \mathrm{s}$ ) and very small optical hysteresis once sufficiently polymer stabilized. Experimental results were explained by a well-fitted phenomenological description based on a simulation of the electric addressing fields and Jones calculus. In the presented model, both a flexoelectro-optic effect and a Kerr-effect were considered in order to describe the electro-optic response of the polymerstabilized cells.

The short pitch chiral nematic LC was prepared by adding 5 wt. \% of high twisting power chiral dopant R5011 to the nematic mixture BL006 (both obtained from Merck $\mathrm{KGaA}$ ). Subsequently, up to 9 wt. $\%$ of a photopolymerizable LC, UCL-011 (obtained from Dainippon Ink \& Chemicals), were added to this chiral nematic LC in order to obtain photopolymerizable chiral nematic LC mixtures. These mixtures were prepared and stored in brown glass containers to avoid unwanted exposure to UV light. These glass containers were stored in an oven at temperature of $130{ }^{\circ} \mathrm{C}$ overnight in order to provide thorough thermal mixing of all contained components. The LC test cells (cell gap of $d=4.6 \mu \mathrm{m}$ ) were filled by capillary action on a hot-plate at temperature of $130^{\circ} \mathrm{C}$ in a yellow-lit environment. These LC test cells (fabricated by LG Displays) possessed patterned in-plane electrodes (Figure 1) with electrode width of $4 \mu \mathrm{m}$ and electrode separation of $10 \mu \mathrm{m}$. The glass surfaces of the standard test cells were coated with rubbed (anti-parallel rubbing in $z$-direction) polyimide alignment layers. Filled test cells were carefully investigated by optical polarized microscopy and showed a homogeneous LC-alignment (Grandjeantexture) with helix axis perpendicular to the glass surfaces (parallel to the $y$-direction). The electro-optic response was measured by using a transmitted light polarized optical microscope fitted with a halogen white light source, $589 \mathrm{~nm}$ interference filter, temperature controlled sample holder, and photodiode-detector (Thorlabs PDA55). The polarizers were crossed and the temperature was set to $30^{\circ} \mathrm{C}$. The test cells were mounted on the sample holder and both polarizers were carefully oriented at an angle of $\pm \pi / 4$ with respect to the electrode direction (Figure 1). Electrical addressing of test cells was conducted with a computer programmed function generator and an in-house built amplifier. Both applied square-wave voltages and detector signal were read with a digital storage oscilloscope. Additionally, microscopic images were recorded with a digital imaging system (MediaCybernetics Evolution MP 5.0). UV-curing of test cells was conducted with an UV curing system (EXFO Omnicure S1000) at a power density of $5 \mathrm{~mW} / \mathrm{cm}^{2}$ and exposure time of $15 \mathrm{~min}$.

The detected optical power as a function of the applied voltage in the neat (without any addition of photopolymerizable LC) chiral nematic LC is shown (Figure 2) along with a micrograph of the test cell recorded after the experiment (Figure 2, inset). Test cells of the neat chiral nematic LC required to be investigated when freshly filled (Figure 2). In the initial state, the LC was homogeneously oriented in the Grandjean-texture and accordingly, an optical extinct initial state was detected. As expected, the detected optical power increased if a voltage was applied. However, without polymer stabilization, the test device showed large hysteresis and the optical extinct state was not restored after the voltage

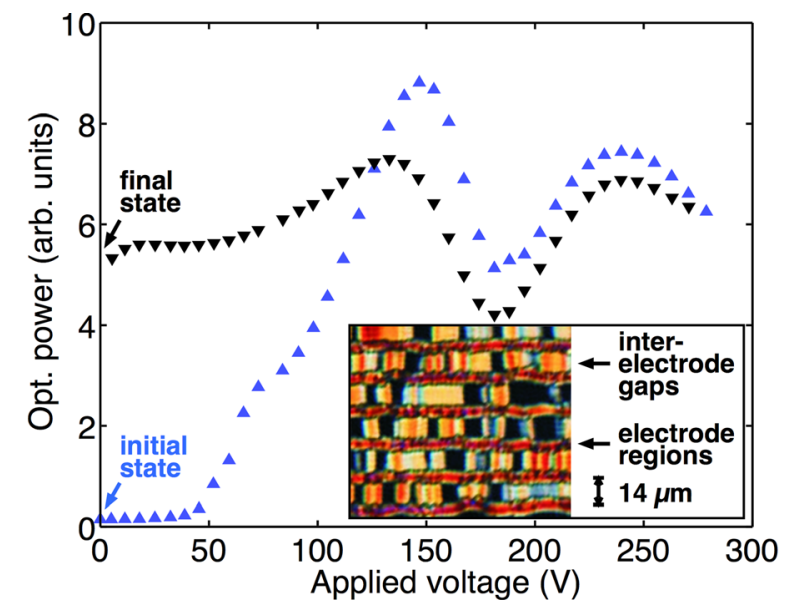

FIG. 2. Electro-optic response (detected optical power vs. applied voltage) of the neat chiral nematic LC recorded with increasing voltage (upright triangles) and decreasing voltage (down-pointing triangles). The inset shows a micrograph of the LC texture in the final state.

reached $0 \mathrm{~V}$ again. The optical micrograph of the test cell after applying a high voltage shows that the LC-layer suffers from unwanted, irreversible textural transitions. ${ }^{23,24}$

In order to understand these textural transitions in more detail, the electric field distribution inside test cells were simulated in a two-dimensional model with COMSOL. ${ }^{25}$ In this simulation, the $4.5 \mu \mathrm{m}$ thick LC-layer was simulated along with $10 \mu \mathrm{m}$ thick sections of adjacent glass plates, for the purpose to allow electric fields to decay within the area of calculation (Figure 3). The chiral nematic mixture BL006 had local dielectric constants of $\varepsilon_{\perp}=4.7$ and $\varepsilon_{\|}=20.0 .^{26}$ However, the LC was straightforwardly modeled as an isotropic material of average dielectric constant $\varepsilon_{L C}=\left(2 \varepsilon_{\perp}+\varepsilon_{\|}\right) / 3$. This approach was instructive to understand the behavior of the LC by means of well-known effects $^{26}$ and thus trace them back to (locally) homogeneous electric fields. Accordingly, the simulated evolution of the electric field distribution with increasing addressing voltage is shown (Figure 4). Even at low addressing voltages, high field strengths were present near the electrode regions. The green contour line corresponds to a field strength of $10 \mathrm{~V} / \mu \mathrm{m}$, which in the investigated mixture corresponds to the critical voltage of the well-known textural transition between Grandjean-texture and focal conic texture. ${ }^{26}$ If this critical field strength is exceeded (by a continuous voltage

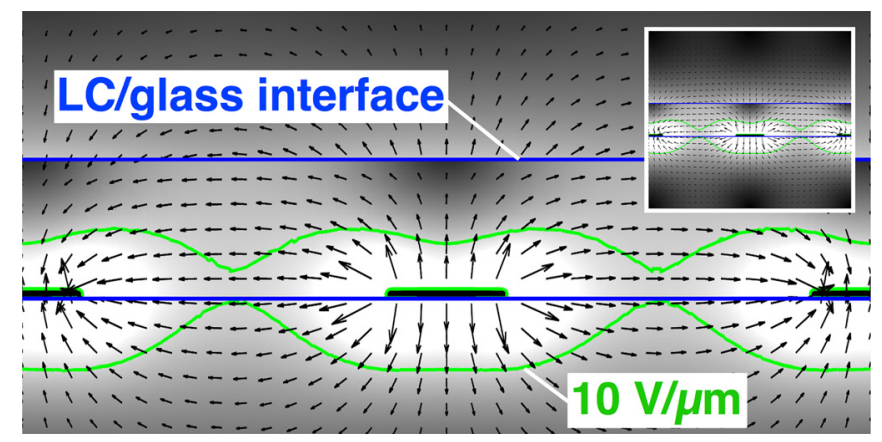

FIG. 3. Simulation result of the electric field distribution inside the test cells at a voltage of $150 \mathrm{~V}$. The complete area of simulation is shown as inset $(24.5 \times 28 \mu \mathrm{m})$. The magnitude of the electric field is indicated as a shaded region and the field direction is plotted as a vector field. 
(a)

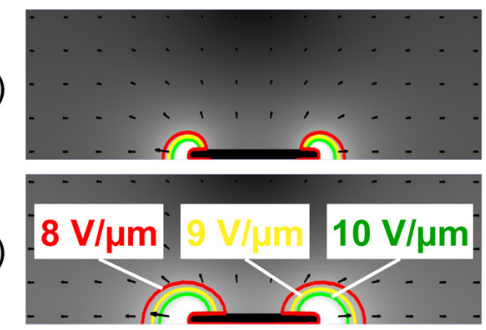

$60 \mathrm{~V}$

(b)

(c)

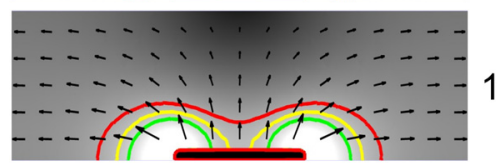

$80 \mathrm{~V}$

$100 \mathrm{~V}$

(d)

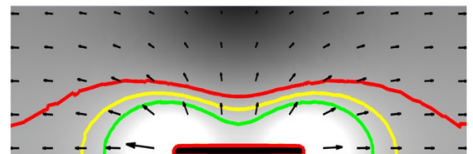

$120 \mathrm{~V}$

(e)

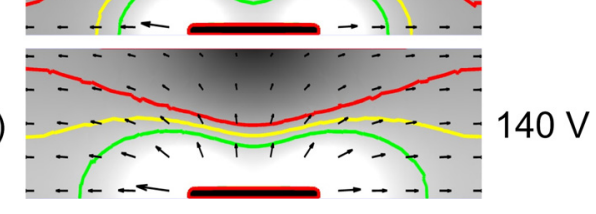

FIG. 4. Simulated electric field distributions near a single electrode caused by applied voltages from (a) $60 \mathrm{~V}$ to (e) $140 \mathrm{~V}$ in steps of $20 \mathrm{~V}$.

ramp), the chiral nematic LC will retain the induced molecular orientation, even after the voltage is switched off again. Such unwanted textural transitions were even likely to occur in polymer-stabilized samples at low concentrations $(<9$ wt. \%) of photopolymerizable LC and especially near the electrode positions (as explained by the high field strength observed in the simulations at these positions).

A photopolymerizable $\mathrm{LC}$ concentration of $9 \mathrm{wt}$. \% was experimentally found to be sufficient to suppress unwanted textural transitions. The electro-optic response of a polymer stabilized test cell was investigated with monochromatic light $(589 \mathrm{~nm}, 60 \times$ objective lens). Microscopic images of the transmitted light were recorded at various applied voltages (Figure 5). The imaging system was carefully calibrated and transmission profiles were extracted from the recorded image files (several periods of interdigitated electrodes were averaged).

In the following, the measured results will be presented along with calculated results that were obtained in a theoretical approach based on Jones calculus. First, the calculated electric field distributions inside the LC-layer were exported from the triangular simulation grid into a rectangular grid with a resolution of 128 data points in $x$-direction and 10 data points in $y$-direction so as to divide the LC-layer into 10 planar slabs. In the next step, two well-known theoretical
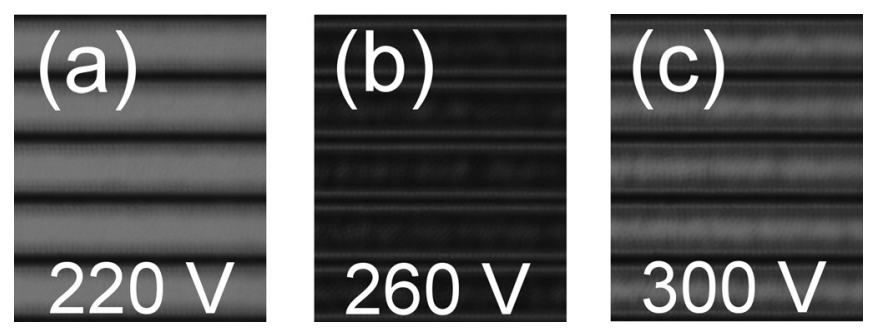

FIG. 5. Microscopic images recorded with quasi-monochromatic light $(589 \mathrm{~nm})$ showing the same region of a polymer stabilized test cell at (a) $220 \mathrm{~V}$, (b) $260 \mathrm{~V}$, and (c) $300 \mathrm{~V}$. expressions (and physical data of BL006 ${ }^{27}$ ) were used to describe locally induced birefringence at each grid point. The first expression was for the flexoelectro-optic response, ${ }^{8}$ where $\theta_{m}$, the maximum induced tilt angle, depended on the electric field component $E_{x}$ (perpendicular to the helical axis), the helical pitch, the splay and bend elastic constants $\left(K_{1}=17.9 \mathrm{pN}, K_{3}=33.5 \mathrm{pN}\right)$, and the flexoelectric coefficient $e_{f}$ of the LC:

$$
\theta_{m}=\arctan \left(\frac{2 e_{f} p E_{x}}{2 \pi\left(K_{1}+K_{3}\right)}\right)
$$

By averaging the ordinary and extraordinary refractive indices $\left(n_{o}=1.530, n_{e}=1.809\right)$ of the LC, the effective refractive index $n_{\text {eff }}$ was obtained:

$$
n_{e f f}=\sqrt{\frac{n_{e}^{2}+n_{o}^{2}}{2}},
$$

As result, the flexoelectro-optically induced birefringence $\Delta n_{f}$ was calculated:

$$
\Delta n_{f}=\left(\left(\frac{\cos \left(\theta_{m}\right)}{n_{e f f}}\right)^{2}+\left(\frac{\sin \left(\theta_{m}\right)}{n_{o}}\right)^{2}\right)^{-1 / 2}-n_{e f f} .
$$

The helical pitch was adjusted to $250 \mathrm{~nm}$ (18 complete helical twists in the LC layer), which was sufficient to avoid selective reflection of visible wavelengths [selective reflection occurs at $\left.\left(\lambda \approx n_{\text {eff }} p\right)\right]$. In addition to the flexoelectrooptic response, a Kerr effect electro-optic response was also considered. Owing to the Kerr effect, birefringence $\Delta n_{K}$ is induced approximately given by:

$$
\Delta n_{K}=\lambda K_{K e r r} E^{2}
$$

where $\lambda$ is the optical wavelength and $K_{K e r r}$ is the Kerr constant.

The optic axis was projected onto the $x$-axis and using a phenomenological description the two values of induced birefringence were summed up to obtain the overall induced birefringence $\Delta n_{i}=\Delta n_{f}+\Delta n_{f}$ at each grid point of the rectangular mesh. Thus, the model was capable of describing each electro-optic response solely (either $e_{f}=0$ or $K_{\text {Kerr }}=0$ ) or as a sum $\left(e_{f}>0\right.$ or $\left.K_{K e r r}>0\right)$. The maximum value of $\Delta n_{i}$ was numerically limited by the birefringence of BL006 $(\Delta n=0.286)$. The local Jones matrices ${ }^{27,28}$ of wave plates with optic axis in the $x$-direction were obtained for each grid point. Subsequently, the transmission of light propagating in the $y$-direction was calculated by Jones calculus for initially unpolarized light that sequentially was transmitted through a linear polarizer, a set of 10 electrically induced wave plates, and a second linear polarizer as analyzer. The polarization directions of polarizer and analyzer were $-\pi / 4$ and $+\pi / 4$, respectively (angles given with respect to the $x$-axis). The obtained high-resolution (128 data points in $x$-direction) transmission profiles are shown (Figure 6(a)) for three voltages. The sharp features of these profiles are unlikely because the optical resolution (which is intrinsically decreased by thermal fluctuations of the LC director) was 


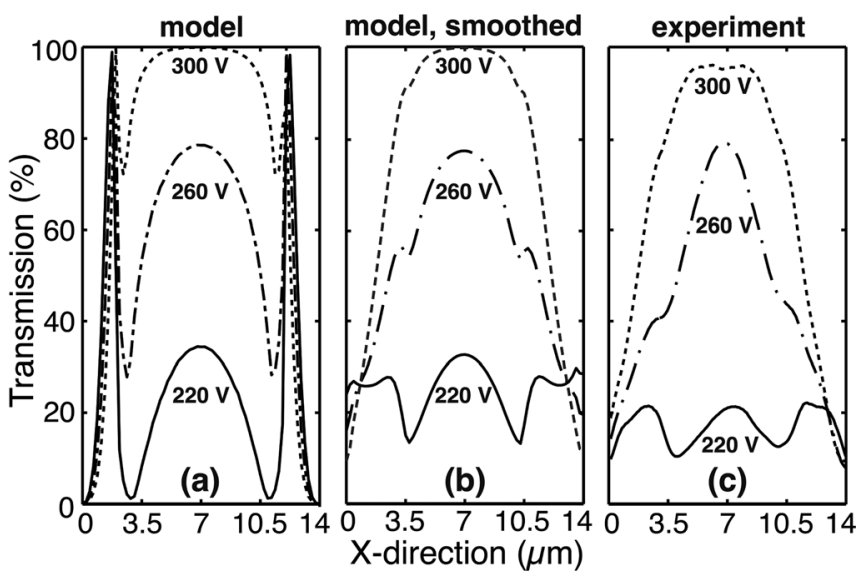

FIG. 6. Estimated transmission profile of a polymer stabilized test cell with high resolution [(a) model], smoothed to a feature size of $2.5 \mu \mathrm{m}$ [(b) model, smoothed], and measured transmission profiles of such a test cell recorded with a $60 \times$ objective lens [(c) experiment].

overestimated. To account for limited resolution, the calculated transmission profiles were smoothed (Figure 6(b)). These smoothed profiles qualitatively fit the experimentally observed ones (Figure 6(c)) very well. In order to obtain a fit that allows for estimates of the coefficients, the average transmission was calculated for each transmission profile (at each voltage) and transmission vs. voltage curves were recorded (with carefully calibrated photodiode detector and $60 \times$ objective lens). This experiment was repeated several times and the transmission vs. voltage curves were averaged in order to extract the experimental error. For values of $e_{f}=30.5 \mathrm{pCm}^{-1}$ and $K_{\text {Kerr }}=1.8 \times 10^{-10} \mathrm{~m} / \mathrm{V}^{2}$, model and experiment (Figure 7(a)) fit within the experimental error bar. In summary, neither of the two electro-optic effects applied in the theoretical model can solely explain the recorded transmission vs. voltage behavior: The flexoelectro-optic response is apparently dominant at low voltages $(<150 \mathrm{~V})$. In contrast, the Kerr effect, where the

(a)

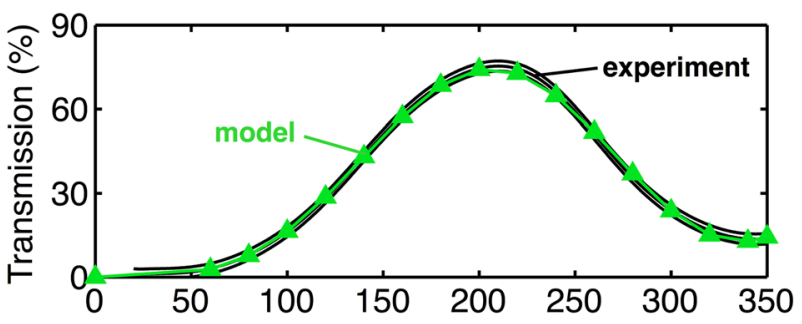

(b)

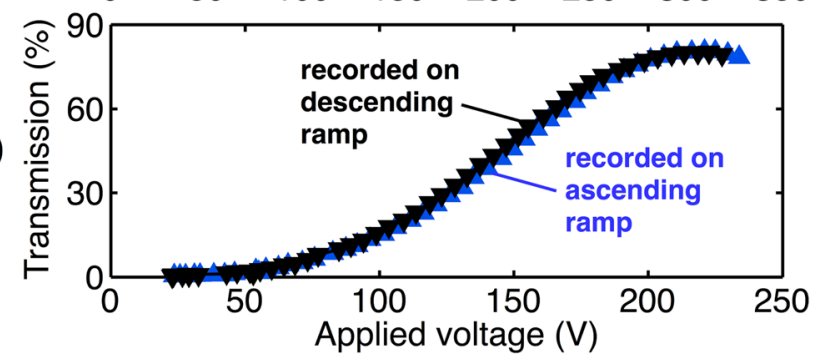

FIG. 7. (a) Transmission data recorded with carefully calibrated photodiodedetector, monochromatic light, and $60 \times$ objective lens on ascending voltage ramps (black double line); double line indicates $2 \sigma$-experimental error obtained on analyzing several experimental runs. Additionally, modeled data is shown. (b) Transmission vs. applied voltage recorded on ascending and subsequently descending voltage ramp. induced birefringence is proportional to $E^{2}$, mainly contributes to the induced birefringence at high voltages.

Very low hysteresis of the electro-optic response was experimentally observed in the stabilized samples (Fig. 7(b)) by applying an ascending and subsequently descending voltage ramp. A maximum voltage of $\approx 230 \mathrm{~V}$ was used, in slight excess of the voltage $(\approx 210 \mathrm{~V})$ required to reach maximum transmission. A short square wave pulse signal ${ }^{12}$ (a train of three bipolar pulses of $\pm 210 \mathrm{~V}$ amplitude) was applied in order to measure the rise and decay times $\left(\tau_{\text {on, }}, \tau_{\text {off }}\right)$ of the transmitted intensity. A 10\%-90\% change of transmitted intensity was considered, respectively. As expected in this USH device, due to the short chiral nematic pitch and polymer network, fast electro-optic response times were experimentally measured $\left[\tau_{\text {on }}=270 \mu \mathrm{s}\left(30^{\circ} \mathrm{C}\right), \tau_{\text {on }}=50 \mu \mathrm{s}\left(60^{\circ} \mathrm{C}\right)\right.$, $\tau_{\text {off }}=280 \mu \mathrm{s}\left(30^{\circ} \mathrm{C}\right)$, to $\left.\tau_{\text {off }}=80 \mu \mathrm{s}\left(60^{\circ} \mathrm{C}\right)\right]$.

In conclusion, the electro-optic response of a fast switching $\left(\tau_{\text {on }}+\tau_{\text {off }} \ll 1 \mathrm{~ms}\right)$ polymer stabilized hyper-twisted chiral nematic liquid crystal was studied and the electro-optic behavior was traced back to and explained by well-known electro-optic effects. Polymer stabilization was found to effectively suppress unwanted textural transitions of the chiral nematic LC especially near the electrodes. The simulation of the inhomogeneous addressing electric fields was very useful to fit and explain the experimental transmission vs. voltage curves. The electro-optic response was mainly caused by the flexoelectro-optic effect at low voltages and by the Kerr effect at high voltages. In addition to the electro-optics of chiral nematic LCs, we believe that the presented approach could be also very useful to investigate other fast electro-optic effects in LCs, such as the electro-optic response of polymer-stabilized blue phase LCs. ${ }^{29,30}$

One of the authors (S.M.M.) acknowledges The Royal Society for financial support. One of the authors (A.L.) acknowledges financial support by the German Research Council (DFG Lo 1922/1-1). W.S.K. and S.S.C. would like to thank Jong-Hoon Woo, Joong-Ha Lee, Dong-Guk Kim, and Woo-Sup Shin of LGD for supporting this work.

${ }^{1}$ J. S. Patel and R. B. Meyer, Phys. Rev. Lett. 58, 1538 (1987).

${ }^{2}$ N. A. Clark and S. T. Lagerwall, Appl. Phys. Lett. 36, 899 (1980).

${ }^{3}$ H. J. Coles, M. J. Clarke, S. M. Morris, B. J. Broughton, and A. E. Blatch, J. Appl. Phys. 99, 034104 (2006).

${ }^{4}$ P. Rudquist, L. Komitov, and S. T. Lagerwall, Liq. Cryst. 24, 329 (1998).

${ }^{5}$ P. Rudquist, M. Buivydas, L. Komitov, and S. T. Lagerwall, J. Appl. Phys. 76, 7778 (1994).

${ }^{6}$ G. Hegde and L. Komitov, Appl. Phys. Lett. 96, 113503 (2010).

${ }^{7}$ B. J. Broughton, M. J. Clarke, R. A. Betts, T. Bricheno, and H. J. Coles, Proc. SPIE 5741, 190 (2005).

${ }^{8}$ F. Castles, S. M. Morris, and H. J. Coles, Phys. Rev. E. 80, 031709 (2009).

${ }^{9}$ F. Castles, S. M. Morris, D. J. Gardiner, Q. M. Malik, and H. J. Coles, J. Soc. Inf. Disp. 18, 128 (2010).

${ }^{10}$ H. H. Lee, J. S. Yu, J. H. Kim, S. I. Yamamoto, and H. Kikuchi, J. Appl. Phys. 106, 014503 (2009).

${ }^{11}$ S. S. Choi, F. Castles, S. M. Morris, and H. J. Coles, Appl. Phys. Lett. 95, 193502 (2009).

${ }^{12}$ D. J. Gardiner, S. M. Morris, F. Castles, M. M. Qasim, W.-S. Kim, S. S. Choi, H.-J. Park, I.-J. Chung, and H. J. Coles, Appl. Phys. Lett. 98, 263508 (2011).

${ }^{13}$ I. Dierking, Adv. Mater. 12, 167 (2000).

${ }^{14}$ P. Archer and I. Dierking, J. Opt. A 11, 024022 (2009). 
${ }^{15}$ B. J. Broughton, M. J. Clarke, S. M. Morris, A. E. Blatch, and H. J. Coles, J. Appl. Phys. 99, 023511 (2006).

${ }^{16}$ G. P. Crawford, S. Zumer, L. C. Chien, F. Vicentini, and Y. Lin, Liquid Crystals in Complex Geometries (Taylor \& Francis, London, 1996).

${ }^{17}$ I. Dierking, L. L. Kosbar, A. Afzali-Ardakani, A. C. Lowe, and G. A. Held, Appl. Phys. Lett. 71, 2454 (1997).

${ }^{18}$ D. K. Yang, L. C. Chien, and J. W. Doane, Appl. Phys. Lett. 60, 3102 (1992).

${ }^{19}$ D. K. Yang, J. L. West, L. C. Chien, and J. W. Doane, J. Appl. Phys. 76, 1331 (1994).

${ }^{20}$ C. V. Rajaram, S. D. Hudson, and L. C. Chien, Chem. Mater. 7, 2300 (1995).

${ }^{21}$ A. Y. G. Fuh, M. S. Tsai, and C. Y. Huang, Jpn. J. Appl. Phys., Part 1 35, 3960 (1996).
${ }^{22}$ D. J. Gardiner, S. M. Morris, P. J. W. Hands, F. Castles, M. M. Qasim, W. S. Kim, S. S. Choi, T. D. Wilkinson, and H. J. Coles, Appl. Phys. Lett. 100, 063501 (2012).

${ }^{23}$ H. Xianyu, S. Faris, and G. P. Crawford, Appl. Opt. 43, 5006 (2004).

${ }^{24}$ S. A. Jewell and J. R. Sambles, Phys. Rev. E. 78, 012701 (2008).

${ }^{25}$ COMSOL Multiphysics ${ }^{\circledR}$, Version 4.3.

${ }^{26}$ D.-K. Yang, X.-Y. Huang, and Y.-M. Zhu, Annu. Rev. Mater. Sci. 27, 117 (1997).

${ }^{27}$ Datasheet of BL006 (Nematel) at a wavelength of $589 \mathrm{~nm}$ and a temperature of $20^{\circ} \mathrm{C}$.

${ }^{28}$ E. Collett, Field Guide to Polarization (SPIE Press, Bellingham, WA, 2005).

${ }^{29}$ H. Kikuchi, M. Yokota, Y. Hisakado, H. Yang, and T. Kajiyama, Nature Mater. 1, 64 (2002).

${ }^{30}$ G. Nordendorf, A. Lorenz, A. Hoischen, J. Schmidtke, H. Kitzerow, D. Wilkes, and M. Wittek, J. Appl. Phys. 114, 173104 (2013). 